\title{
Metal-ceramic joining by laser
}

\author{
J.M. PELLETIER and M. ROBIN
}

GEMPPM-CALFETMAT, Bat. 403, INSA-Lyon, 69621 Villeurbanne cedex, France

\begin{abstract}
Within the scope of increase in cutting tools efficiency, a $\mathrm{CW} \mathrm{CO}_{2}$ laser beam has been used for butt welding sintered tips containing cobalt and tungsten carbide to a medium carbon steel core. After optimization of the laser processing conditions, full penetrating, sound and homogeneous welds are observed. Hardness is found to be nearly constant across the entire weld bead cross-section. Micrographic observations in the same area show a fine dendritic microstructure ; microanalysis (EDS) as well as X-ray diffraction experiments reveal that dendrites contain mainly iron and cobalt, while tungsten has segregated in the interdendritic zone where complex (Fe - Co - W) rich carbides are likely to form.
\end{abstract}

\section{I- Introduction}

Improvement of the performances of steels has often to be improved, since many components are used in severe environments : corrosion or wear are often involved and, even, at elevated temperature. Tools used for machining are typical examples. A solution may be retained : addition of a very hard tip, either mechanically clamped, brazed or welded (1). Among many techniques, laser welding has different advantages : flexibility, limited affected zone and, therefore, only small deformations are induced.

The present study will then be devoted to laser welding of cemented carbide tips. Substrates are medium carbon steels. A binding element, cobalt-base material, is added to the carbides, in order to facilitate the mixing.

\section{II - Experimental procedure:}

For laser welding, core is made of a medium carbon steel with the following composition (wt \%): $0.35 \% \mathrm{C}-1 \% \mathrm{Cr}-0.2 \%$ Mo - bal.Fe. Parallelepipeds $\left(50 \times 25 \times 2 \mathrm{~mm}^{3}\right)$ were cut from oil quenched and tempered plates. Tips contain cobalt $(20 \%)$ and tungsten carbide $(80 \%)$. They are processed by a powder-metallurgy technique and cut in parallelepipeds $\left(40 \times 8 \times 3.2 \mathrm{~mm}^{3}\right)$. Only $W C$ and Co phases are detected by $X$ ray diffraction prior to welding. Core and tip sides are polished and cleaned prior to welding. Parts are then abutted without gap and mechanically clamped together.

Specimen were mounted on a numerically controlled $X-Y$ table and irradiated by a continuous $\mathrm{CO}_{2}$ laser, of power up to $3.6 \mathrm{~kW}$. The laser beam was focused by a spherical $\mathrm{Zn}$ Se lens (focusing distance: $127 \mathrm{~mm}$ ). Scanning rate $V_{T}$ of the sample under the laser beam 
ranged from 0.2 to $3 \mathrm{~m} / \mathrm{mn}$. A variation of the interaction time, defined by $t=d / V_{T}$, where $d$ is the equivalent beam diameter on the sample, was thus possible. During scanning, argon was blown through a nozzle, in order to prevent oxidation.

For observations using optical or scanning electron microscopy and for Vickers hardness measurements, specimen were cutted and polished (down to $1 \mu \mathrm{m}$ ). For optical micrography, etching was finally performed. Different reagents were used : Murakami and Struers V2A for coatings, tool steel and stainless steel, Nital for mild steel.

A 840 A JEOL microscope was used for scanning electron microscopy; either backscattered or secondary electrons were taken to form the image (BEI or SEI mode, respectively). The same apparatus was equipped with an EDS microanalysis system (Tracor).

$X$ ray diffaction patterns were obtained with a $\mathrm{Cu}(\mathrm{KX})$ radiation and a graphite monochromator.

\section{III - Experimental results for laser welding:}

A good weld should meet at least the following quality features : full penetration, structural homogeneity and no apparent defect (holes, cracks) either in the weld bead or in the heat affected zone (HAZ).

Different defects are always present during preliminary attempts. Big holes are often observed in the central part of the weld and cracks appear in the weld bead as well as in the $H A Z$ on the steel side. Finally, we have found more convenient to slightly defocuse the beam in order to enlarge the interaction zone and to shift the beam towards the steel side, in order to first melt the steel and make the melt pool progress more easily into the tip.

The weld bead is homogeneous and a full penetration is achieved. Four different zones are always detected in cross-sections, as illustrated in figure 1 ; from the top to the bottom, we observe:

a - the base steel (HV $\approx 350-400)$ : microstructure is typical of an oil quenched and tempered medium carbon steel, i.e. a fine tempered martensite revealed by etching.

b - the steel $H A Z(H V \approx 400-450)$ : as in other laser treated steels (2), classical microstructures can be recognized :

- a martensitic microstructure, near the weld bead; here, a high peak temperature has induced full austenitization upon heating and rapid quenching is achieved by efficient heat transfer to cold steel.

- a bainitic microstructure in various conditions :

. either far from the weld bead. As shown by lon et al (3), the diffusion of carbon atoms concerns only very limited distances and a complete dissolution of carbides is therefore impossible. Austenitizing is only partial and volume fraction of martensite able to form during cooling is small.

. or because cooling rate is too low, as reported by Pergue et al (4), after laser melting of a medium carbon steel $(0.48 \%)$.

c - the weld bead $(H V \approx 1000)$

This microstructure is mainly dendritic, in spite of some irregular aspect. These irregularities can be attributed to different factors :

- owing to a cross-section bias effect of a three-dimensional growth network, dendrites appear to be randomly oriented;

- convection flow is produced in the melted pool due to Marangoni effect (5-7). Therefore, temperature gradients exist at the beginning of the solidification phenomena, with different orientations. So solidification is not initiated from a plane interface with an uniform temperature gradient, but on the opposite, boundaries are irregular, directions are nearly random and rates are high.

d - the tip (HV $\approx 900-1000$ )

The spatial repartition of carbides is fairly regular. 
Distribution of the different elements in the various zones has been determined by EDS. Due to its low atomic mass, carbon was not detected. Contents of the different elements in the weld bead are as follows (Fig.2) : $70 \% \mathrm{Fe}, 25 \% \mathrm{~W}, 3 \% \mathrm{Co}$ (wt \%). So, iron is the main element present in the melt.

Optical microscopy has revealed the dendritic microstructure of the weld bead ; scanning electron microscopy confirms this result (Fig. 3). In addition, a chemical contrast appears : tungsten, a heavy element, is bright, while lighter elements ( $\mathrm{Fe}, \mathrm{Co}, \mathrm{Cr}, \mathrm{Mo}, . .$.$) are$ grey or dark. Therefore, the inner part of the dendrites contains mainly $\mathrm{Fe}$ and $\mathrm{Co}$, while $W$ is mainly rejected in the interdendritic zones. However, it cannot be concluded that $\mathrm{Fe}$ or $\mathrm{Co}$ are not present in these zones; it is only sure that their concentration is smaller here than within dendrites.

Starting from experimental evidence, the bonding mechanism may be described as follows : the laser beam interacts first with the steel part and induces its melting; the liquid steel wets the cobalt present in the tip and yields rapid melting of this element; convective flow originating in the melt carries the WC particles far away from the cermet boundary and enhances their dissolution rate. An homogeneous liquid phase containing $\mathrm{Fe}, \mathrm{Co}, \mathrm{W}$ and $\mathrm{C}$ is thus obtained. After laser-material interaction, cooling begins and solidification starts with the formation of Fe-Co rich dendrites, while the segregating element $(W)$ is rejected in the interdendritic zones. Fe-Co equilibrium phases diagram (8) shows that solubility of $\mathrm{Co}$ in $\mathrm{Fe}$ is fairly large and even complete at high temperature; it may thus be assumed that dendrites are constituted by an austenitic Fe-Co solid solution, at least at high temperature. As for the interdendritic liquid, its solidification occurs in a second step and yields probably complex carbides $(\mathrm{Fe}, \mathrm{Co}, \mathrm{W})_{\mathrm{x}} \mathrm{C}_{\mathrm{y}}$.

\section{v-Conclusion:}

After various attempts and optimization of the processing parameters - laser power, scanning speed, defocusing distance... - good laser welding has been achieved, in spite of the very different properties (especially their melting point) of the materials : a medium carbon steel core and a tungsten carbide-rich material.

The weld bead $(H V \approx 1000)$ has a dendritic microstructure, which is likely to be obtained in two main steps. First, formation of an homogeneous $\mathrm{Fe}-\mathrm{Co}-\mathrm{W}$ - C liquid solution after cobalt wetting and melting by liquid steel and dissolution of WC particles stirred into the melt by convection. Second, solidification leading to a Fe-Co rich solid solution and $W$ rich mixed carbides in interdendritic areas.

\section{References :}

1 - QUIGLEY M.B.C., in "Physics of Welding", J.F. Lancaster ed., Pergamon Press (1986), p. 306

2 - ASHBY M.F. and EASTERLING K.E., Acta Metall., 32 (1984) 1935.

3 - ION J.C. , ASHBY. M.F. and EASTERLING K.E., Acta Metall., 32 (1984) 1949.

4 - PERGUE D. PELLETIER J.M. , FOUQUET F. and MAZILLE H., J. Mat. Sci., 24 (1987) 4343.

5 - MARANGONI C., Ann. Phys. Chem., 143 (1871) 337.

6 - CHAN C., MAZUMDER J. and CHEN M., Met. Trans., A15 (1984) 2175.

7 - GALERIE A., PONS M. and CAILLET M., Mat. Sci. Eng., 88 (1987) 127.

8 - MASSALSKI T.B., "Binary Phase Diagrams", ASM, Metals Park, Ohio (1986). 


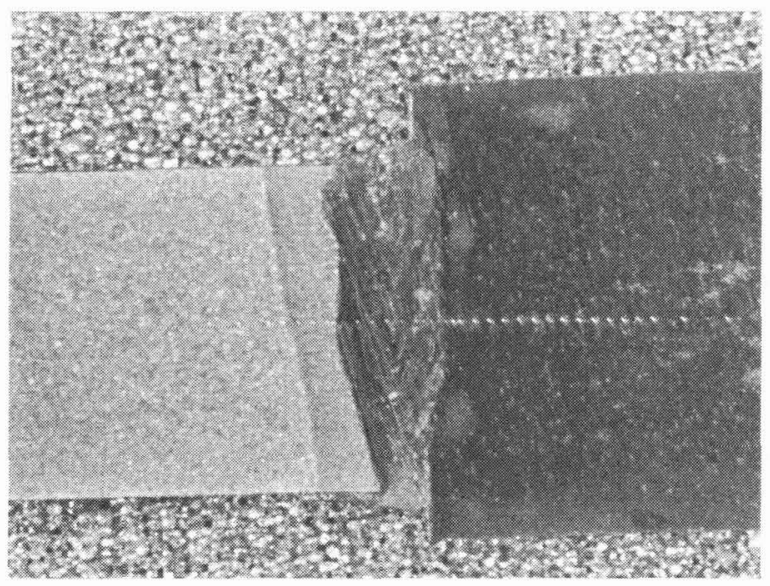

Fig. 1 : typical micrography of a sound weld ; $P=2040 \mathrm{~W} ; \mathrm{vT}=2 \mathrm{~m} / \mathrm{mn} ; \mathrm{d}=0.4 \mathrm{~mm}$; shift of the beam towards the steel side : $\delta=0.3 \mathrm{~mm}$.

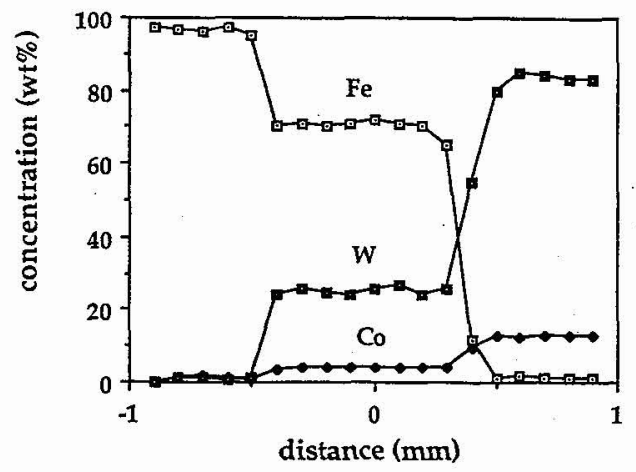

Fig. 2 : concentration profiles, determined by EDS, of the different elements in the various areas of the sample shown in Fig. 1.

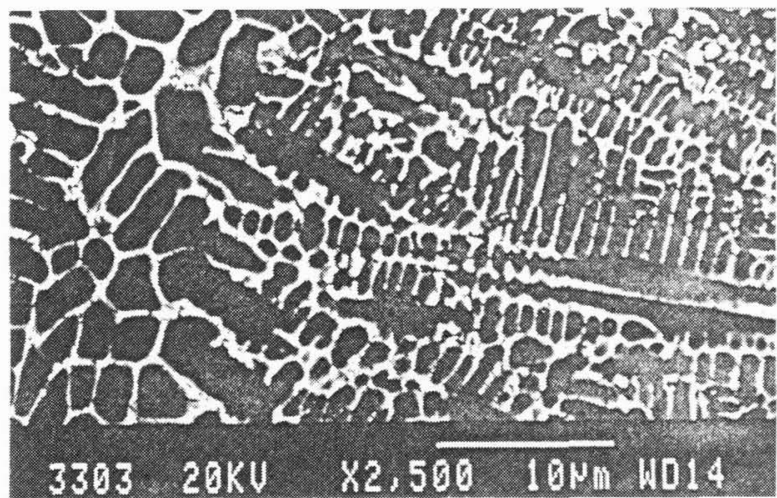

Fig. 3 : dendritic microstructure of the melted zone shown in Fig. 1; scanning electron micrography (BEI mode). 